\title{
EL ORIGINAL MANUSCRITO \\ DE LA COMEDLA DE FERNANDO DE ROJAS: UNA CONJETURA
}

\author{
Germán Orduna ( $\dagger$ ) \\ Universidad de Buenos Aires \\ SECRIT-CONICET
}

En este año de 1999 se conmemoran los 500 años de la primera edición conocida de la Comedia de Calisto y Melibea. Dejando de lado las dudas que el colofón rehecho de Fadrique de Basilea pudiera plantear, es justo celebrar el medio milenio de la aparición en impreso de la más extraordinaria creación en prosa de fines de la Edad Media y comienzo inimitable del lenguaje dramático en lengua española.

En esta ocasión me ha parecido conveniente apuntar algunas reflexiones sobre la curiosa afirmación que cierra la 'Carta del auctor a vn su amigo':

E por que conozcais donde comiençan mis mal doladas razones e acaban las del antiguo auctor: en la margen hallareys vna cruz: e es en fin de la primera cena. Vale.

Es bien sabido que este mismo lugar de la Carta se modifica en la versión de la Tragicomedia, donde leemos:

E por que conozcais donde comiençan mis mal doladas razones, acorde que todo lo del antiguo autor fuesse sin diuision en vn auto: o cena inclusa hasta el segundo auto donde dize: Hermanos mios, etc. 
En esta segunda redacción, el autor se remite a un lugar en el texto donde puede leerse "Hermanos míos, " palabras de Calisto que inician el acto segundo que hoy conocemos.

Sabemos que la edición de 1499 carece del folio inicial en el ejemplar conservado. La afirmación de la Carta en el texto de la Comedia procede de la editada en Toledo, en 1500, primer ejemplar que da el texto de la Carta, lo que muestra la persistencia en el texto impreso, de una realidad que corresponde evidentemente a un texto manuscrito.

Si la única manera de advertir dónde comienza la continuación de Fernando de Rojas es por una cruz puesta en el margen, cabe suponer que al redactar la 'Carta del auctor a vn su amigo,' Rojas tenía ante sí un texto manuscrito que se iba a transmitir por copias, en las cuales "en la margen hallareys vna cruz" era el único medio para reconocer el comienzo del texto de Rojas.

Es curioso que las ediciones posteriores de la Comedia mantuvieran ese rasgo de la tradición manuscrita y sólo en la Carta de la Tragicomedia se lo enmendara en forma que permite suponer un texto impreso. Que sólo una cruz al margen se utilice para separar la contribución de Rojas permite también presuponer un manuscrito continuo y abigarrado donde las separaciones de un acto a otro estaban poco marcadas o no se dejaban espacios que permitieran hacer visible esa separación.

En la "Carta" de la Comedia leemos: "hallareys vna cruz: e es en fin de la primera cena. Vale."; lo que permite inducir que Rojas pensaba en la Comedia organizada en escenas. Al menos habría una primera 'çena' muy extensa, constituida por el texto del anónimo autor y una segunda 'çena' que comienza donde hay una cruz al margen, lugar que se indica más claramente en la Carta enmendada para la Tragicomedia:

acorde que todo lo del antiguo autor fuesse sin diuision en un auto o cena inclusa hasta el segundo auto donde dize: Hermanos mios, etc.

Explícitamente habla en la Tragicomedia del "segundo acto," que quedaba implícito en la Carta publicada en la Comedia: "e es en fin de la primera cena"; lo que supone otras 'cenas' o autos. En el llamado Prólogo, que Rojas agrega después de las Octavas Acrósticas en la edición de la Tragicomedia, leemos:

quien negará que aya contienda en cosa que de tantas maneras se entienda: que avn los impressores han dado sus punturas poniendo rúbricas o sumarios al principio de cada 
auto narrando en breve lo que dentro contenía [...]. (Valencia, 1514, Aiiii)

Podemos ver en esto que en cuanto a la inclusión de los argumentos, el mismo Rojas nos dice que es obra de los impresores, con lo que podemos inferir por un lado, que la división en autos estaba hecha en la Comedia manuscrita, pero no resultaba fácilmente visible en el texto manuscrito como después lo era, por la inclusión de los argumentos, en el texto impreso. El ejemplar conservado, impreso por Fadrique de Basilea tenía ya los argumentos que precedían a cada auto y además, un grabado hacía evidente la separación en autos; de ninguna manera es esta la disposición textual a que alude Rojas en la 'Carta del autor a vn su amigo.'

La edición de la Comedia (Toledo, 1500) sólo tiene un grabado en la portada y la separación de los autos se hace evidente por la inclusión de los argumentos y la enumeración de los personajes que intervienen en cada auto.

Pareciera como si la 'Carta del auctor a vn su amigo' que Rojas escribió para la edición de la Comedia manuscrita no fue revisada por Fernando de Rojas al hacerse las impresiones en letras de molde.

En lo que va de nuestro análisis podemos adelantar como observación provisoria que cuando Fernando de Rojas escribía la Carta como preliminar de la Comedia manuscrita, ésta era un texto continuado en el que sólo alguna leve indicación - como podría ser la enumeración de los personajes que intervenían en el auto - servía de indicador del comienzo de un nuevo acto. De otra manera no hubiera sido necesaria la inclusión de una cruz en el margen; además, esos actos carecían del argumento previo que Rojas nos dice que incluyeron los impresores.

La tradición manuscrita de la Comedia ha sido puesta de manifiesto con la publicación en 1990 del ms. de la Biblioteca de Palacio $\mathrm{n}^{\circ} 1520$, hecho por Charles B. Faulhaber [Celestinesca 14.2, 3-39). La escuela de Roma, que trabaja desde hace más de una década en la edición crítica del texto de la Comedia y el de la Tragicomedia, era la destinataria inmediata del descubrimiento publicado en 1990. Patrizia Botta y Francisco Lobera han trabajado intensamente sobre el fragmento de Palacio y publicaron sendos estudios en el Boletín de la Real Academia Española en 1993, a lo que se sumó el facsímil del manuscrito. En años posteriores, uno y otro han vuelto sobre el tema con aproximaciones más afinadas. En 1997 se publicaron en Valencia las comunicaciones a unas Jornadas sobre el tema - «Cinco siglos de Celestina: aportaciones interpretativas» -, entre las que destacamos a nuestro propósito las de Patrizia Botta y Juan Carlos Conde. 
El consenso general destaca la importancia del manuscrito de Palacio $1520(=M p)$ para la restitución del texto de la Comedia: sus lecciones manifiestan un texto de un estadio anterior y con excelentes datos frente a los tres conocidos hasta 1990; es decir, el estado de los tres impresos: el de Burgos, $1499(B)$, Toledo, $1500(C)$ y Sevilla, $1501(D)$. A estos estudios minuciosos, en los que se aplica la mejor técnica ecdótica, quiero ahora agregar algunas observaciones siguiendo el enfoque que he adoptado en lo anteriormente expuesto.

Cuando Francisco Lobera estudia en 1993 "El manuscrito 1520 de Palacio y la tradición impresa de La Celestina, "advierte que el texto que nos transmite $M p$ es muy próximo al del texto de la Comedia transmitido en las tres ediciones conocidas, "pero con numerosísimas variantes léxicas y, sobre todo, sintácticas y estilísticas, desplazamientos, supresiones y añadidos que, en su conjunto, no pueden ser simples variantes de copia, sino que a todas luces son variantes de redacción."

Juan Carlos Conde, en su trabajo de 1997, ofrece también, en las conclusiones, un balance del cotejo de Palacio 1520 con la tradición impresa: "el alto número de errores de copia, puramente mecánicos, las deficiencias en la transcripción de nombres propios y muchos otros defectos que se alternan en nuestro manuscrito, con lecturas llamativas por su calidad textual hacen preciso postular una gran vitalidad en esta rama de la tradición textual de la Celestina, rama que podemos considerar sin grave riesgo de error representación de una fecunda - aunque breve en el tiempo, desplazada pronto por la tradición impresa - transmisión de la obra, de la cual sólo nos queda este representante" (185).

Lobera al estudiar $M p$ y la tradición impresa toca otro tema importante en la consideración del manuscrito de Palacio y apunta una serie de propuestas sobre la procedencia del ms.: (1) Pueden ser "papeles del antiguo autor" de que habla Rojas (autógrafo o su copia); (2) copia derivada del auto del antiguo autor, diferente de los papeles que llegaron a Rojas; (3) un autógrafo - borrador del texto de Rojas; (4) una copia revisada por Rojas; (5) una copia más o menos mediata de la Comedia de Rojas (en una versión no definitiva); (6) copia de una edición perdida (no descripta, es decir, de una rama distinta de las ediciones conocidas); o (7) copia de una de las ediciones de la Comedia o la Tragicomedia que ha llegado hasta nosotros. Las cuatro primeras propuestas han sido desechadas por la crítica; las tres restantes están en evaluación.

En la conferencia escrita por Lobera y Botta para el Seminario de Soria (1995)titulada "El texto de Celestina" se vuelve a presentar el problema del ms. 1520 y los niveles redaccionales conjeturables en el proceso de génesis 
de la obra. Botta supone que $M p$ es representante de una Comedia originaria (la llama Ur-Celestina), de tradición manuscrita, con rasgos distintos del texto de tradición impresa.

Sin entrar en el amplio marco de datos con que se lleva adelante la discusión sobre el problema de la ubicación estemática del manuscrito de Palacio, queremos aportar esta nuestra línea de trabajo que se basa en los tres lugares aducidos al principio, es decir, la primera forma y la corrección hecha en la Carta, la alusión a la intervención de los impresores en la redacción del argumento de cada acto, y la inspección del manuscrito de Palacio.

Parece oportuno recordar que el manuscrito de Palacio aparece transcrito por dos manos y termina fragmentariamente al final de uno de los folios de copia, en pleno parlamento de Pármeno, cuando describe a Calisto la vieja casa de Celestina. Botta sostiene (1997) que todos los indicios apuntan a que el manuscrito de Palacio, originalmente, contenía la Comedia completa, que en esa fase textual no incluía la Carta ni los Acrósticos.

El texto manuscrito comienza después de un anagrama del nombre de Cristo (Ihs), que era comienzo corriente de una copia, después del cual se lee:

Síguese la Comedia de Calisto y Melibea conpuesta en reprehension de los locos enamorados que vençidos en su deshordenado apetito a sus amigas llaman y dizen ser su dios. Asimesmo fecha en aviso de los engaños de las alcauetas y malos y lisonjeros seruientes:

A este epígrafe sigue el conocido argumento general. Se incluye el encabezamiento, Argumento, y se lee:

Calisto fue de noble linaje, de claro ingenio, de gentil disposición, de linda criança, dotado de muchas gracias, de estado mediano. Fue preso en el amor de Melibea, muger moça, muy generosa, de alta y sereníssima sangre, sublimada en próspero estado, una sola heredera a su padre Pleberio, y de su madre Alisa muy amada. Por solicitud del pungido Calisto, vencido el casto propósito della - entreveniendo Celestina, mala y astuta muger, con dos servientes del vencido Calisto, engañados y por ésta tornados desleales, presa su fidelidad con anzuelo de codicia y de deleyte vinieron los amantes y los que les ministraron en amargo y desastrado fin.

Concluye el Argumento con estas palabras que merecen nuestra atención: 
Para comienço de lo qual dispuso el adversa fortuna lugar oportuno, donde a la presencia de Calisto se presentó la desseada Melibea.

A continuación en una misma línea se escribe: "Calisto y Melibea. comiença Calisto" y a continuación, en el folio siguiente: "Calisto. en esto veo Melibea la grandeza de Dios." Y continúa por 7 folios el texto conocido del Aucto I hasta el final fragmentario en el parlamento de Pármeno.

Obsérvese cómo el argumento general termina con una frase que introduce el insólito y brusco comienzo del acto primero ("En esto veo Melibea la grandeza de Dios") con una redacción que elude toda referencia espacial ("dispuso el aduersa fortuna lugar oportuno donde a la presencia de Calisto se presentó la deseada Melibea"). Podrá conjeturarse que el mismo Rojas escribió el Epígrafe y el Argumento general como textos introductorios apropiados para el comienzo "in medias res" del auto primero.

La serie de observaciones que hemos realizado nos lleva a una posición próxima a la propuesta de Botta (1997), quien supone que el manuscrito de Palacio es muestra de una fase manuscrita de la obra en que circuló totalmente anónima (y no semi-anónima como la fase impresa de la Comedia), sin prólogos del autor ni epílogos.'

Por nuestra parte, sostenemos que el Epígrafe y Argumento General que hemos comentado poco antes, corresponden a la fase manuscrita, y son obra de Rojas.

Es oportuno recordar aquí la opinión expuesta por Marcel Bataillon en 1961 en su importante estudio, La CélestineselonFernandode Rojas, donde analiza agudamente los textos liminares de la Comedia y conjetura que el folio perdido de la edición de Burgos 1499, pudo contener el Epígrafe y el Argumento General, y señala el mismo final del Argumento que hemos destacado antes ("donde a la presencia de Calisto se presentó la deseada Melibea") y se pregunta Marcel Bataillon: "Ceci ne témoignerait-il pas l'état primitif du texte, où les arguments particuliers de chaque acte n'existaient pas?"

Y a continuación, como en este trabajo mismo se hizo independientemente, Bataillon acude al texto del Prólogo incluido en la Tragicomedia, donde Rojas se refiere a la intervención de los impresores en la inclusión de los argumentos de cada acto, lo que Rojas reprueba como recurso

\footnotetext{
${ }^{1}$ Agradezco a Patrizia Botta su rápida respuesta a mis requerimientos bibliográficos.
} 
no utilizado por los antiguos escritores. Nada dice en cambio Rojas del Argumento General porque sí era común incluirlo. También Stephen Gilman en un trabajo especial sobre los argumentos de Celestina (1954) hizo observaciones coincidentes y llegó a pensar que el 'Argumento de toda la obra' había sido escrito por el primer autor anónimo. Lo curioso es que Bataillon que, como es obvio, no pudo conocer $M p$, llega a conjeturar la existencia de una impresión anterior a la de Burgos donde el Argumento General apareciera encabezando la edición, pero sin los argumentos de cada acto.

Como Bataillon y Gilman, he comenzado por dar crédito a las aserciones de Fernando de Rojas en los paratextos que aparecen en la tradición impresa de la Comedia; pero nos sorprende la remisión a una señal al margen como posibilidad de reconocer el comienzo del acto segundo de la Comedia, sobre todo cuando en la versión de la Tragicomedia se corrige la referencia con indicación que remite ya a un texto impreso.

Nos interrogamos sobre esta anomalía de que una Carta agregada al texto impreso parezca remitir a un texto de presentación manuscrita. El testimonio reciente del manuscrito de Palacio agrega nuevos elementos de juicio que nos permiten proponer las siguientes conclusiones:

1. Rojas no intervino en la tradición impresa de la Comedia de la que proceden Toledo, $1500(C)$ y Sevilla, $1501(D)$, quizás tampoco en Burgos, sobre la que no abrimos juicio por falta de los textos liminares;

2. $\quad M p$ copia un texto que se inicia con el epigrafe ("Siguese la Comedia") y el argumento general; prescinde del argumento del acto primero y entra directamente al diálogo poniendo: "Calisto y Melibea. comiença Calisto";

3. La disposición del texto de $M p$ no se corresponde con la tradición impresa;

4. Rojas achaca a los impresores el "poner rúbricas y sumarios al principio de cada auto"; pero nada dice del Argumento General;

5. El Argumento General introduce directamente al diálogo dramático aludiendo al lugar ("dispuso la adversa fortuna lugar oportuno"), lo que podría atribuirse a un estadio primitivo de la Comedia (coincidimos en esto con Gilman y Bataillon). Conjeturamos que pueda ser obra de Rojas que vio en el Argumento una manera de introducir el parlamento inicial de Calisto; 
6. El $M p$ reflejaría el estado primitivo del texto en su fase manuscrita (Epígrafe + Argumento General + diálogo de comienzo ex-abrupto). Coincidimos en esto con Botta; $y$

7. Rojas es el autor del Epígrafe y el Argumento General;

He querido exponer brevemente estas mis reflexiones sobre este asunto tan interesante y dejar espacio para la posible intervención de colegas especializados en Celestina que seguramente nos enriquecerán con sus propias reflexiones.

\section{REFERENCIAS BIBLIOGRÁFICAS}

Bataillon, Marcel, 1961. "La Célestine" selon Fernando de Rojas. Paris, Didier, esp. p. 15.

Botta, Patrizia, 1993. "La Celestina de Palacio en sus aspectos materiales. I," Boletín de la Real Academia Española (enero-abril 1993), 25-50.

_, 1993. "La Celestinade Palacio en sus aspectos materiales (cont.)," ibid (mayo-agosto 1993), 347-367.

, 1995. "Ancora sulla genesi e paternità de La Celestina, "Cultura Neolatina, 55, 269-283.

- 1997. "El texto en movimiento (de La Celestina de Palacio a La Celestina posterior)" (Actas del Curso de Valencia, 25-29 marzo, 1996), en Cinco Siglos de Celestina: Aportacionesinterpretativas, ed. R. Beltrán \& J. L. Canet. Valencia, Universidad, 135-159.

Conde, Juan Carlos, 1997. "El manuscrito II-1520 de la Biblioteca de Palacio y La Celestina: balance y estado de la cuestión", en Cinco Siglosde Celestina: Aportacionesinterpretativas, ed. R. Beltrán \& J. L. Canet. Valencia, Universidad, 161-185.

Gilman, Stephen, 1954. "The 'Argumentos' to La Celestina," Romance Philology 8.2, 71-78 (incluido en el Apéndice B de The Art of 'La Celestina', Madison: U Wisconsin P, 1956, 212-216).

Lobera Serrano, Francisco J., 1993. "El manuscrito 1520 de Palacio y la tradición impresa de La Celestina, "Bol. de la Real Academia Española (enero-abril 1993), 51-67. 